EPJ Web of Conferences 116, 04006 (2016)

DOI: $10.1051 /$ epjconf/201611604006

(C) Owned by the authors, published by EDP Sciences, 2016

\title{
KM3NeT/ARCA sensitivity and discovery potential for neutrino point-like sources
}

\author{
A. Trovato ${ }^{\mathrm{a}}$ on behalf of the KM3NeT Collaboration \\ INFN-LNS, via S. Sofia 62, 95123 Catania, Italy
}

\begin{abstract}
KM3NeT is a large research infrastructure with a network of deep-sea neutrino telescopes in the abyss of the Mediterranean Sea. Of these, the KM3NeT/ARCA detector, installed in the KM3NeT-It node of the network, is optimised for studying high-energy neutrinos of cosmic origin. Sensitivities to galactic sources such as the supernova remnant RXJ1713.7-3946 and the pulsar wind nebula Vela X are presented as well as sensitivities to a generic point source with an $\mathrm{E}^{-2}$ spectrum which represents an approximation for the spectrum of extragalactic candidate neutrino sources.
\end{abstract}

\section{Introduction}

KM3NeT is a new Research Infrastructure, consisting of a network of deep-sea neutrino telescopes in the Mediterranean Sea. In response to the IceCube discovery of cosmic neutrinos [1] and the reported nonzero value for the neutrino mixing angle $\theta_{13}$, KM3NeT 2.0 will consist of two detectors with different granularity: KM3NeT/ARCA [2] at the KM3NeT-It site (Capo Passero, Italy) with two building blocks dedicated to high-energy neutrino astronomy and KM3NeT/ORCA [3], a single building block located at the KM3NeT-Fr site (Toulon, France), dedicated to the study of the neutrino mass hierarchy. Thanks to its geographical location in the Northern hemisphere, KM3NeT/ARCA can observe most of the Galactic Plane, including the Galactic Centre. The detection of galactic point sources is one of the main goals of the experiment. Under the hypothesis of $100 \%$ hadronic mechanisms acting inside transparent sources, models for galactic neutrino emission are well constrained by $\mathrm{TeV}$ gamma-ray observations and allow the calculation of realistic predictions of the detector discovery potential.

Neutrinos are detected by measuring the Cherenkov light induced by charged secondary particles emerging from neutrino interactions. KM3NeT will consist of arrays of light detectors deployed in the deep, clear water of the Mediterranean Sea which acts as target, Cherenkov radiator and shield for atmospheric muons. The light detectors are photo-multiplier tubes (PMTs) arranged in glass spheres that resist the water pressure (digital optical modules, DOM [4]). The optical modules are arranged along flexible strings with a total height of about $700 \mathrm{~m}$ for KM3NeT/ARCA. This detector will consist of two building blocks of 115 strings each, with 18 DOMs per string and a spacing of $36 \mathrm{~m}$ between

\footnotetext{
a e-mail: atrovato@lns.infn.it
}

This is an Open Access article distributed under the terms of the Creative Commons Attribution License 4.0, which permits unrestricted use, distribution, and reproduction in any medium, provided the original work is properly cited. 
DOMs. The footprint will be roughly circular with an average distance between strings of about $90 \mathrm{~m}$. Each block will have a volume of about $\sim 0.5 \mathrm{~km}^{3}$.

\section{Simulation codes and analysis}

The software used in this work [5] provides a complete simulation for neutrinos of all flavours with energy in the range $10^{2}-10^{8} \mathrm{GeV}$, including their interaction in the medium $[6,7]$, the propagation of the emerging secondary particles [8], the light generation and propagation in water and the detector response. The depth and the optical water properties measured at the KM3NeT-It Capo Passero site are used [9]. Background light due to the presence of ${ }^{40} \mathrm{~K}$ in salt water has been also simulated.

The neutrino induced events can be divided into two classes, track-like and cascade-like events, each class requiring a specific event reconstruction. Only the results of the track analysis will be reported here. The reconstruction algorithm for track-like events used in this work is described in $[10,11]$. The angular resolution, calculated as the median angle between the reconstructed muon direction and the generated neutrino track, is about $0.2^{\circ}$ at $10 \mathrm{TeV}$.

In point source searches, the simulated events are analysed through statistical techniques to look for an excess around the source position. Indeed, the neutrino signal from a cosmic source needs to be identified among the large background of atmospheric muons and neutrinos, produced by the interaction of primary cosmic rays with the atmosphere. The atmospheric neutrino event rate is calculated assuming the Honda et al. [12] conventional atmospheric model, the Enberg et al. [13] prompt component and adding a correction due to the cosmic rays knee on the neutrino spectrum as described in [14]. Atmospheric muons are generated with the MUPAGE event generator [15]. Atmospheric muons can be rejected by selecting only upward-going events, which can be only produced by neutrinos, the only particles that can traverse the whole Earth without being absorbed. This rejection requires a reliable determination of the zenith angle. For this reason beside a zenith angle cut, a cut on the goodness of fit criterion of the reconstruction is applied. Atmospheric neutrinos are an unavoidable background but a key for a possible discrimination against cosmic neutrinos is their different spectrum, which is softer than the expected spectrum of cosmic neutrinos.

The discovery potential, that is the signal flux required to obtain an observation at a given significance level (e.g. $5 \sigma$ or $3 \sigma$ ) with $50 \%$ probability [16], has been taken as a figure of merit of the telescope performance. The method used considers two hypotheses: the hypothesis that data consist only of background and the hypothesis that signal events are present in the data in addition to the background. The likelihood of obtaining the data given each hypothesis is calculated, and the ratio of likelihoods, or equivalently the $\log$ of the likelihood ratio, serves as test statistic [11, 17]. Having Probability Density Functions (PDF) that describe the distribution of signal and background events as a function of a given variable $x, P D F_{\text {sig }}(x)$ and $P D F_{b k g}(x)$, the likelihood ratio can be written as:

$$
L R=\sum_{i=1}^{n} \log \frac{\frac{n_{\text {sig }}}{n} \times P D F_{\text {sig }}\left(x_{i}\right)+\left(1-\frac{n_{\text {sig }}}{n}\right) \times P D F_{b k g}\left(x_{i}\right)}{P D F_{b k g}\left(x_{i}\right)}
$$

where $n$ is the total number of recorded events in a given period of time and $n_{\text {sig }}$ is the expected number of signal events in the sample of $n$ events. For each sample $L R$ is maximised as function of $n_{\text {sig }}$ and the maximum value of $L R$ is used as test statistic. The variable $x$ in eq. 1 is chosen in different ways in the analyses described in the following and will be discussed in each case.

\section{Results}

The operation of gamma-ray telescopes have revealed in the last decades a large number of $\mathrm{TeV} \gamma$-ray sources in our Galaxy associated with Supernova Remnants (SNR) and Pulsar Wind Nebulae (PWN) 

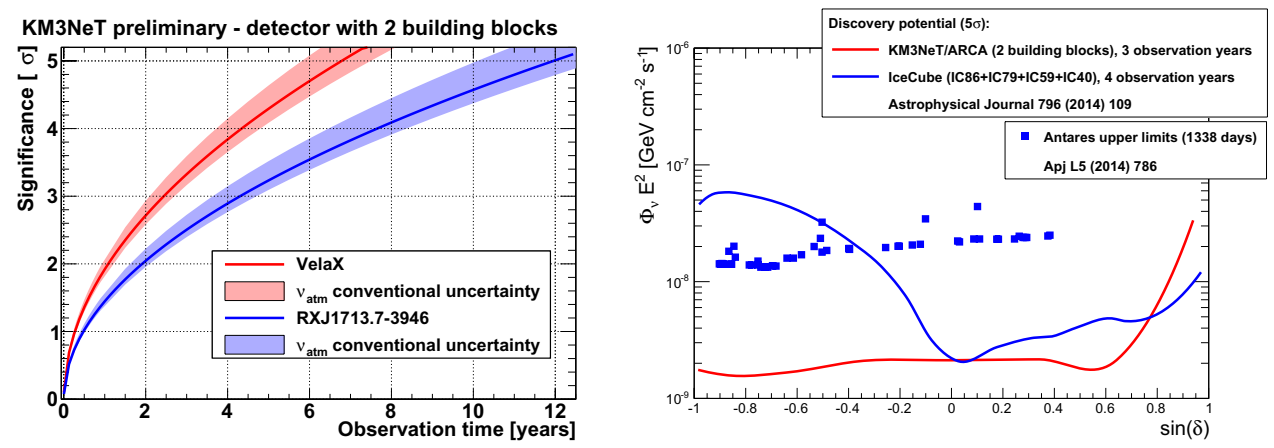

Figure 1. Left: Significance of the RXJ1713.7-3946 (blue line) and Vela X (red line) observation as a function of observation time for the KM3NeT/ARCA detector. Right: KM3NeT/ARCA $5 \sigma$ discovery flux (red line) per flavor for point sources with an $E^{-2}$ spectrum as a function of their declination calculated for 3 years of observation time. For comparison also the IceCube 4 years discovery flux [23] and the upper limit for the ANTARES detector [24] are shown.

that are also promising neutrino sources. In particular, the SNR RXJ1713.7-3946 and the Pulsar Wind Nebulae (PWN) Vela X are at present two of the most intense galactic objects in the high-energy gammaray band and are used here to evaluate the KM3NeT/ARCA performance. In both cases the expected neutrino spectra are calculated from the measured $\gamma$-ray spectrum under the hypotheses of a transparent source and $100 \%$ hadronic emission.

The young shell-type SNR RX J1713.7-3946 has been observed by H.E.S.S. [18] and its gamma energy spectrum is measured up to about $100 \mathrm{TeV}$. Having a declination of $-39^{\circ} 46^{\prime}$ it is visible by KM3NeT for about $80 \%$ of the time. This SNR has been simulated as an extended homogeneous neutrino source with a $0.6^{\circ}$ radius and an energy spectrum parametrised as [19]:

$$
\Phi(E)=16.8 \times 10^{-15}\left[\frac{E}{\mathrm{TeV}}\right]^{-1.72} e^{-\sqrt{E / 2.1 T e V}} \mathrm{GeV}^{-1} \mathrm{~s}^{-1} \mathrm{~cm}^{-2} .
$$

Vela $\mathrm{X}$ is one of the nearest PWN centered at RA $=08^{h} 35^{m}$, DEC $=-45^{\circ} 36^{\prime}$. The VHE $\gamma$-ray emission from a circular region of $0.8^{\circ}$ around the source centre is reported by H.E.S.S. [20]. The corresponding neutrino spectrum derived using the Vissani prescription [21] is parametrised as:

$$
\Phi(E)=7.2 \times 10^{-15}\left[\frac{E}{\mathrm{TeV}}\right]^{-1.36} e^{-(E / 7 \mathrm{TeV})} \mathrm{GeV}^{-1} \mathrm{~s}^{-1} \mathrm{~cm}^{-2} .
$$

The source has been simulated as a flat spatial distribution within a disk of $0.8^{\circ}$ radius.

Under the assumed hypotheses, the significance of the observation as a function of the observation time for KM3NeT/ARCA has been calculated and is shown in the left panel of Fig. 1. In both cases a Boosted Decision Tree (BDT) from the ROOT TMVA package [22] has been used and the distributions of the BDT output for the signal and background events are used as PDFs in Eq. (1). An observation with $3 \sigma$ significance is expected with the KM3NeT/ARCA detector after about 2.5 and 4.5 years for Vela X and RXJ1713.7-3946, respectively. The major uncertainty in this calculation is due to the contribution of conventional component of the neutrino flux. This uncertainty, shown as a shaded area in Fig. 1, is taken into account assuming a $\pm 25 \%$ variation in the normalization of the Honda et al. flux model [12].

The discovery flux is also evaluated for a generic $E^{-2}$ point source as an approximation for neutrino extragalactic sources. The right panel in Fig. 1 shows the $5 \sigma$ discovery flux as a function of the point source declination. In this case the PDFs in Eq. (1) are functions of the angular distance of the reconstructed track from the source and the number of hits, $N_{h i t}$, in a space-time correlation with the 
fitted track. The parameter $N_{\text {hit }}$ is a rough energy estimator. The 3 years observation time has been chosen in such a way to have a comparable exposure with respect to the IceCube results in [23]. The different trend of the KM3NeT and IceCube curves is mainly due to the geographical location of the detectors. KM3NeT can reach an unprecedented sensitivity in the Southern hemisphere and good sensitivity everywhere else compared to IceCube.

\section{Conclusions}

The results presented in this paper represent the status of the point source analysis and show that at least the most intense galactic ones are within reach for KM3NeT. In particular, the expectation for RXJ1713.7-3946 and Vela X are reported. The discovery potential for point sources with $E^{-2}$ spectrum shows that KM3NeT has a very large field of view thus not only complementing, but also overlapping to a large extent the IceCube one. KM3NeT can thus provide important contributions to the new born field of neutrino astronomy.

\section{References}

[1] M.G. Aartsen et al. (IceCube), Phys. Rev. Lett. 113, 101101 (2014)

[2] R. Coniglione et al. (KM3NeT), These proceedings (2015)

[3] D. Samtleben et al. (KM3NeT), These proceedings (2015)

[4] R. Bruijn et al. (KM3NeT), PoS(ICRC15)1157 (2015)

[5] A. Margiotta et al. (ANTARES), NIM A 725, 98 (2013)

[6] G. Ingelmann, A. Edin and J. Rathsman, Comput. Phys. Commun. 101, 108 (1997)

[7] G. Barr, Ph.D. thesis, University of Oxford (1987)

[8] P. Antonioli et al., Astropart. Phys. 7, 357 (1997)

[9] G. Riccobene et al., Astropart. Phys. 27, 1 (2006)

[10] A. Trovato et al. (KM3NeT), PoS(ICRC15)1114 (2015)

[11] A. Trovato, Ph.D. thesis, Università degli Studi di Catania (2014)

[12] M. Honda et al., Phys. Rev. D 75, 043006 (2007)

[13] R. Enberg, M.H. Reno and I. Sarcevic, Phys. Rev. D 78, 043005 (2008)

[14] M.G. Aartsen et al. (IceCube), Phys. Rev. B 89, 062007 (2014)

[15] Y. Becherini et al., Astropart. Phys. 25, 1 (2006)

[16] G.C. Hill, J. Hodges, B. Hughey, A. Karle and M. Stamatikos, Proceedings of PHYSTAT05 p. 108 (2006)

[17] J. Braun et al., Astropart. Phys. 29, 299 (2008)

[18] F. Aharonian et al. (H.E.S.S.), Astron. Astroph. 464, 235 (2007), erratum in A\&A 531, 2 (2011)

[19] S.R. Kelner, F.A. Aharonian and V.V. Bugayov, Phys. Rev. D 74, 063007 (2006)

[20] F. Abramowsky et al. (H.E.S.S.), Astron. Astrophys. 548, A38 (2012)

[21] F.L. Villante and F. Vissani, Phys. Rev. D 78, 103007 (2008)

[22] A. Hoecker et al., PoS ACAT 040 (2007), arXiv: physics/0703039

[23] M.G. Aartsen et al. (IceCube), Astrophys. J. 796, 109 (2014)

[24] S. Adrían-Martínez et al. (ANTARES), Astrophys. J. Lett. L5, 786 (2014) 